\title{
The accuracy of sentinel node mapping according to $T$ stage in patients with gastric cancer
}

\author{
Igor Rabin ${ }^{1}$, Bar Chikman ${ }^{1}$, Ron Lavy ${ }^{1}$, Natan Poluksht ${ }^{1}$, Zvi Halpern ${ }^{1}$, Ilan Wassermann ${ }^{1}$, \\ Ruth Gold-Deutch ${ }^{1}$, Judith Sandbank ${ }^{2}$, and Ariel Halevy ${ }^{1}$ \\ ${ }^{1}$ Division of Surgery, Assaf Harofeh Medical Center, Zerifin 70300, Israel, affiliated to the Sackler Faculty of Medicine, Tel Aviv University, \\ Tel Aviv, Israel \\ ${ }^{2}$ Institute of Pathology, Assaf Harofeh Medical Center, Zerifin 70300, Israel, affiliated to the Sackler Faculty of Medicine, Tel Aviv \\ University, Tel Aviv, Israel
}

\begin{abstract}
Background. Sentinel lymph node (SLN) mapping has been recently introduced to the field of gastric cancer. To the best of our knowledge, no study has dealt with the accuracy of SLN mapping according to the $T$ stage of the primary tumor. The aim of the present study was to evaluate SLN status according to the $\mathbf{T}$ stage of the primary tumors.

Methods. Eighty patients with gastric cancer underwent SLN mapping with patent blue dye during gastric resection.

Results. Forty-seven patients underwent distal subtotal gastrectomy; 17 patients, proximal gastrectomy; 14,total gastrectomy; and 2, gastric stump resection. SLNs were stained in $61 / 80$ patients $(76.3 \%)$. The number of stained SLNs varied from 1 to 16 (mean, 3.3). Patients undergoing proximal gastrectomy had a mean of 3 stained SLNs, whereas patients undergoing distal subtotal gastrectomy had a mean of 2.8 stained SLNs. In 55/61 patients $(90.2 \%)$ with stained SLNs a positive correlation was found between the presence of metastases and stained or non-stained SLNs. Ten out of 11 patients (90.9\%) with T1 tumors (mean, 3.27 SLNs per patient) and 15/17 patients with T2 tumors $(88.2 \%$; mean, 3 SLNs per patient) had stained SLNs as compared to only $33 / 48(68.8 \%)$ of patients with T3 tumors (mean, 3.3 SLNs per patient). The positive predictive value of the SLN mapping was $100 \%$, the negative predictive value was $76.9 \%$, and sensitivity was 85.4\%.

Conclusion. While in $\mathrm{T} 1$ and $\mathrm{T} 2$ tumors sentinel node mapping may be of assistance in the decision-making process regarding the extent of lymphadenectomy (sensitivity, 100\%; negative predictive value, $90 \%-100 \%$ ), SLN mapping in patients with T3 tumors will be misleading in a third of the patients and should not be attempted.
\end{abstract}

Key words Gastric cancer · Sentinel lymph node mapping · Gastrectomy

Offprint requests to: A. Halevy

Received: June 11, 2009 / Accepted: November 26, 2009

\section{Introduction}

The history of sentinel lymph node (SLN) mapping dates back to 1977 when Cabanas [1] described and used the technique of lymphangiograms in patients with penile carcinoma. However, this technique was not practiced for many years until it was reintroduced by Morton and coworkers [2] in the treatment of patients with early-stage malignant melanoma. Since then, sentinel node biopsy has been used in the management of a variety of cancers in the hope that it will eliminate many unnecessary lymph node dissections, resulting in less morbidity. Today, SLN mapping is recognized as a multidisciplinary surgical approach to the management of patients with cutaneous melanoma and breast cancer [3-6], but it is also being evaluated and used in patients with gastrointestinal malignancies, especially gastric cancer [7-12]. The regional lymph node status is crucial for staging and estimating the prognosis of the individual patient with gastric cancer resected for cure [13]. Focused examination of SLNs based on serial sections and immunohistochemistry further increases staging accuracy.

SLN mapping in patients with gastric cancer has been practiced for the past 10 years [7-12]. Several techniques for the administration of dye or radioactive tracer injection have been reported: (a) preoperative endoscopic injection of dye or radioactive tracer followed by intraoperative mapping [7, 13-15]; (b) intraoperative endoscopic injection [12, 16-18]; and (c) intraoperative subserosal injection of dye [11, 19-21]. We adopted the intraoperative technique of open dye injection. The aim of this study was to learn the accuracy of SLN mapping in different $\mathrm{T}$ stages of patients with gastric cancer and to evaluate SLN status according to the $\mathrm{T}$ stage of the primary tumor. 


\section{Patients and methods}

This study was approved by the Institutional Review Board and informed consent was waived.

\section{Patients}

Eighty patients with gastric cancer with no evidence of metastatic disease on a preoperative computed tomography (CT) scan underwent SLN mapping during gastrectomy.

\section{Surgical and staining techniques}

Surgery started with exploration of the abdominal cavity, disease staging, and resectability assessment. Before any dissection was performed, patent blue (Guerbet Patent Blue V Sodium 2.5\%; Guerbet, Roissy, France), diluted with $2 \mathrm{ml}$ of normal saline was injected subserosally in four different opposing points adjacent to the tumor site. Ten minutes following the dye injection, dye spread was evaluated and blue nodes were marked by a stitch. The type of resection was based on tumor location and extent of the disease.

\section{Pathological evaluation}

A detailed focused pathological assessment was performed with special attention to all areas marked by patent blue. All blue-stained lymph nodes were sectioned into $0.2-\mathrm{cm}$-thick slices. Two 3- $\mu \mathrm{m}$-thick sections were serially cut at $0.25-\mathrm{mm}$ levels from these lymph node slices: the first was stained with hematoxylin and eosin and the second was placed on a Superfrost Plus Slide (Menzel GmbH \& Co KG, Braunschweig, Germany). If the H\&E slides were negative for metastatic involvement, the unstained consecutive slides were stained with a pancytokeratin antibody (CKMNF116; Dako, Carpinteria, CA, USA), to highlight micrometastases. All relevant sections were examined. The total sampling of the SLNs with systematic serial sectioning and cytokeratin immunohistochemistry enabled a relatively optimal estimation of the metastatic status of the SLNs.

The non-stained (not sentinel) lymph nodes (LNs) were routinely submitted either in toto, when less than $0.2 \mathrm{~cm}$ in diameter, or sectioned into $0.2-\mathrm{cm}$-thick slices. Two levels of 3- $\mu \mathrm{m}$ thickness were performed on each of these tissue fragments, which were then stained with $\mathrm{H} \& \mathrm{E}$ only.

\section{Results}

\section{Patients}

Eighty patients (27 women and 53 men) with gastric carcinoma and no evidence of metastatic disease underwent SLN mapping during gastric resection. The age range was 26 to 88 years (mean, 67.4 years; median, 70 years). In 30 patients, the tumor was located in the lower third of the stomach; in 24 patients, in the middle third; and in 21, in the upper third or in the gastroesophageal junction -3 patients had linitis plastica and 2 patients had a gastric stump carcinoma following subtotal gastrectomy for benign disease performed many years previously. Forty-seven patients underwent distal subtotal gastrectomy, 17 patients underwent proximal gastrectomy, 14 patients underwent total gastrectomy and 2 patients underwent gastric stump resection, one of them with en-bloc transverse colon resection.

All patients had adenocarcinoma, 26 of them with a signet ring cell component. Fifty-three patients had poorly or moderate to poorly differentiated adenocarcinoma, while 27 patients had well-differentiated adenocarcinoma.

\section{Pathological evaluation}

Altogether, 1539 regional lymph nodes were harvested (mean, 19.2 lymph nodes per patient; median, 18). In 61 of the 80 patients $(76.3 \%)$, blue-stained SLNs were detected and examined, varying from as few as 1 to as many as 16 (mean, 3.3 nodes per patient; median, 2; Fig. 1).

In 61/80 (76.3\%) patients, SLNs were detected during the operation. In this particular group, the mean number of SLNs was 3.3. The mean number of SLNs for the group of patients who underwent proximal gastrectomy $(n=13)$ was 3 , while it was only 2.8 for patients who underwent distal subtotal gastrectomy $(n=35 ; P>0.05$, not significant). These differences were not related to the extent of lymphadenectomy: the mean number of removed LNs in the group of patients who underwent proximal gastrectomy was 18.17 as compared to 18.1 for patients who underwent distal gastrectomy (Table 1).

Table 1. Sentinel lymph node staining, and type of surgical procedure

\begin{tabular}{lcc}
\hline Type of operation & $\begin{array}{c}\text { Overall number } \\
\text { of removed } \\
\text { nodes (mean) }\end{array}$ & $\begin{array}{c}\text { Number of } \\
\text { SLNs (mean) }\end{array}$ \\
\hline Distal gastrectomy & 18.1 & 2.8 \\
Proximal gastrectomy & 18.2 & 3 \\
Total gastrectomy & 25.1 & 5 \\
\hline
\end{tabular}

SLNs, sentinel lymph nodes 
An absence of staining was correlated with the local extension of the primary tumor (Table 2).

The correlation between finding metastatic deposits in these SLNs and in the remainder of the removed lymph nodes in the same patients showed this method to have a high accuracy rate. The accuracy rate (positive correlation with pathological findings) was as high as $90.2 \%$ (55/61 patients). In 35 patients, metastases were found both in stained and non-stained nodes and in 20 patients, there were no metastases found in either stained or non-stained nodes. In 6 patients, metastases were found only in non-stained nodes.

Table 2. Detection rates of stained lymph nodes according to stage of primary tumor

\begin{tabular}{lccc}
\hline Stage & $\begin{array}{c}\text { Number of patients } \\
\text { with stained nodes }\end{array}$ & Percentage & $\begin{array}{c}\text { Mean number } \\
\text { of SLNs }\end{array}$ \\
\hline T1 & $10 / 11$ & 90.9 & 3.27 \\
T2 & $15 / 17$ & 88.2 & 3 \\
T3 & $33 / 48$ & 68.8 & 3.3 \\
\hline
\end{tabular}

SLNs, sentinel lymph nodes
The overall positive predictive value was $100 \%$; the negative predictive value was $100 \%$ for $\mathrm{T} 1,90 \%$ for $\mathrm{T} 2$, and only $50 \%$ for $\mathrm{T} 3$ tumors. A subdivision into $\mathrm{T}$ stages is shown in Table 3.

The data on the six patients with negative SLNs, but positive non-stained nodes are shown in Table 4.

\section{Exclusion from the study}

Four patients were excluded from the study: two patients with carcinoma in situ, because we expected no LN involvement; and two patients with T4 carcinoma (one of them with metastatic peritoneal spread), because we thought we would be unable to glean any contributory data.

\section{Discussion}

Nodal involvement in gastric cancer is defined by two main systems [13]: the American Joint Committee on Cancer/International Union against Cancer (AJCC/ UICC) staging system, which is based on the number of

Table 3. Sensitivity, specificity, positive predictive value, negative predictive value, and overall sentinel lymph nodes vs positive sentinel lymph nodes ratio

\begin{tabular}{lccccc}
\hline Stage & $\begin{array}{c}\text { Sensitivity } \\
(\%)\end{array}$ & $\begin{array}{c}\text { Specificity } \\
(\%)\end{array}$ & $\begin{array}{c}\text { PPV } \\
(\%)\end{array}$ & $\begin{array}{c}\text { NPV } \\
(\%)\end{array}$ & $\begin{array}{c}\text { SLNs vs SLNs(+) } \\
(\text { ratio })\end{array}$ \\
\hline T1 & 100 & 100 & 100 & 100 & $9: 1$ \\
T2 & 83.3 & 100 & 100 & 90 & $7.5: 1$ \\
T3 & 86.2 & 100 & 100 & 50 & $1.6: 1$ \\
\hline
\end{tabular}

PPV, positive predictive value; NPV, negative predictive value; SLNs, sentinel lymph nodes; SLNs(+), positive sentinel lymph nodes
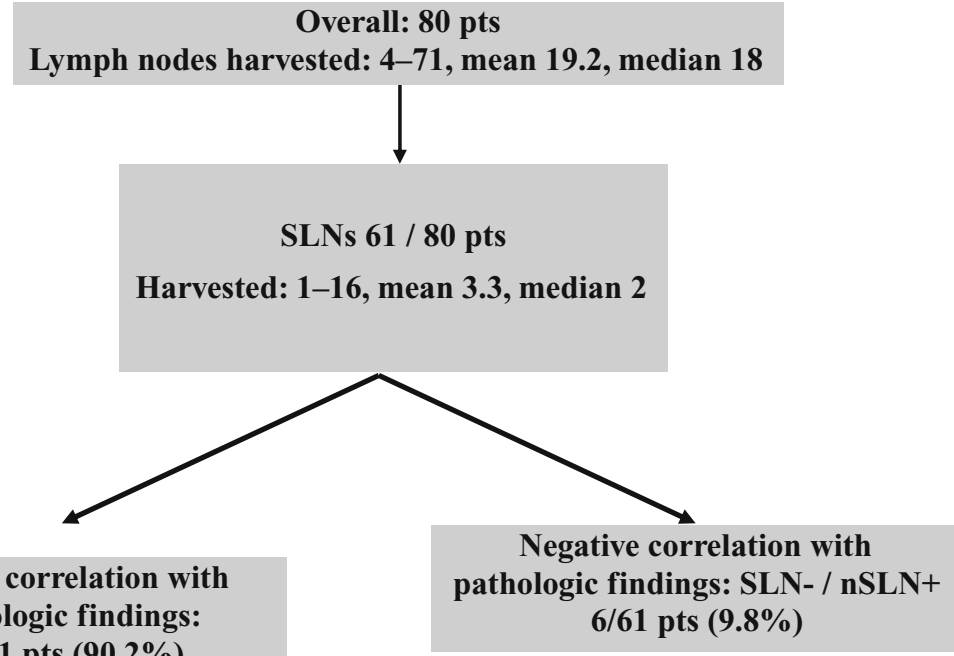

pathologic findings:

$55 / 61$ pts $(90.2 \%)$

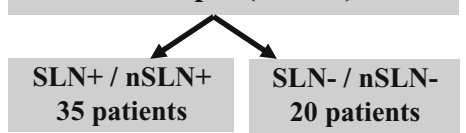

Fig. 1. Results of sentinel node mapping in 80 patients (pts) with gastric cancer. $S L N$, Sentinel lymph node; $S L N+$, indicates metastases in SLNs; $S L N-$, indicates no metastases in SLNs; $n S L N+$, indicates metastases in non-SLNs; $n S L N-$, indicates no metastases in non-SLNs 
Table 4. Data on the six false-negative sentinel lymph node cases

\begin{tabular}{|c|c|c|c|c|c|c|}
\hline $\begin{array}{l}\text { Patient } \\
\text { no. }\end{array}$ & $\begin{array}{l}\text { Location of } \\
\text { tumor }\end{array}$ & $\begin{array}{l}\text { Operation } \\
\text { performed }\end{array}$ & $\begin{array}{l}\text { Overall } \\
\text { LNs }\end{array}$ & $\begin{array}{c}\text { Overall positive } \\
\text { LNs }\end{array}$ & $\begin{array}{l}\text { Overall } \\
\text { SLNs }\end{array}$ & $\begin{array}{l}\text { Location } \\
\text { of SLNs }\end{array}$ \\
\hline 1 & GEJ & PG & 15 & 7 & 1 & 1 \\
\hline 2 & LP & TG & 71 & 62 & 1 & 1 \\
\hline 3 & LT & $\mathrm{DG}$ & 32 & 1 & 2 & 3 \\
\hline 4 & LT & $\mathrm{DG}$ & 12 & 2 & 2 & 6 \\
\hline 5 & LT & $\mathrm{DG}$ & 23 & 2 & 1 & 6 \\
\hline 6 & MT & $\mathrm{DG}$ & 25 & 3 & 5 & 3,4 \\
\hline
\end{tabular}

GEJ, gastroesophageal junction; LP, linitis plastica; LT, lower third; MT, middle third; PG, proximal gastrectomy; TG, total gastrectomy; DG, distal gastrectomy; LNs, lymph nodes; SLNs, sentinel lymph nodes

${ }^{a}$ Number of station according to Japanese classification of regional gastric lymph nodes

positive nodes [22]; and the Japanese system, which is based on the location of positive nodes [23].

The ideal lymphadenectomy for gastric cancer should accurately stage the extent of disease, predict prognosis, and have the potential to improve survival. The rationale for SLN mapping in gastric cancer is twofold: to learn about the extent of the disease (lymph node involvement), and to try to assess the degree to which SLN mapping might aid in the decision-making process regarding the type of gastrectomy to be performed limited or extended [24]. This question has been a matter of debate for years, as Western literature claims no superiority of an extended lymph node dissection and attributes the extent of this dissection to the added morbidity and mortality [25]. We should, however, remember that SLN mapping may mainly affect the extent of lymph node dissection, and to a lesser degree gastric resection.

Various techniques have been used to map SLNs in patients with gastric cancer. Kitagawa et al. [7] used preoperative endoscopic mapping by radioactive tracer; Miwa [16], Nimura et al. [17], and Osaka et al. [18] used endoscopic dye injection; Bilchik et al. [19] and Hiratsuka et al. [20] used intraoperative (open) subserosal injection of dye; and Aikou et al. [14] used injection of radioactive tracer in combination with blue dye injection. As previously stated, we decided to adopt the intraoperative technique of open dye injection.

Reviewing the relevant data, it becomes evident that the success rate of SLN mapping varies from as high as $99 \%$ in the Hiratsuka et al. [20] series or $95 \%$ in the Aikou et al. series [26] to as low as $74 \%$ in other series. Our results show a success rate of approximately $76 \%$.

The application of sentinel node tactics in gastric cancer has been controversial because the number of reported series dealing with this issue is small and the number of patients reported in each of these series is limited. No definitive conclusion can be made regarding the contribution of SLN mapping to the decision-making process regarding the extent of resection. However, some of the current Japanese surgeons still tailor the extent of their dissection according to the result of sen- tinel node mapping, especially for early-stage gastric cancer [27].

We would like to draw attention to some interesting points emerging from our study: The first is in regard to the number of SLNs, which is probably organ-specific owing to the structure and anatomy of the stomach and regional lymphatic network. Gastric lymph channels are multidirectional and form a complex network [28]. Hence, in gastric cancer we are probably not dealing with one SLN, but rather with groups of nodes [22]. It is interesting to note that we were able to localize SLNs in $76 \%$ of our patients, the number of SLNs varying from 1 to 16 , with a mean of 3.3 nodes per patient. We found that the mean number of SLNs was slightly higher in the group of patients who underwent proximal gastrectomy ( 3 nodes per patient) compared to the patients who underwent distal subtotal gastrectomy (2.8 nodes per patient; $P>0.05$, not significant). These differences were unrelated to the extent of lymphadenectomy (Table 1).

The second point is that the decision regarding the type of resection should be based, at least nowadays, on tumor location and $\mathrm{T}$ stage of the tumor until a full evaluation of SLN mapping results is available. However, it seems that the decision can be assisted by SLN mapping.

A third point is an observation that has been reported elsewhere regarding SLN mapping and that has also emerged from our study. We found an inverse correlation between the $\mathrm{T}$ stage of the tumor and the SLN staging. Of the 61 patients with stained nodes, 10 of 11 patients with T1 tumors $(90.9 \%)$ had stained nodes, while this number decreased to $88.2 \%(15 / 17)$ in patients with T2 tumors and to $68.8 \%$ (33/48) in patients with $\mathrm{T} 3$ tumors. We can clearly see that the overall specificity and positive predictive value were $100 \%$ in all T stages, while the negative predictive value decreased from $100 \%$ for $\mathrm{T} 1$ to $50 \%$ for $\mathrm{T} 3$ tumors. We could not find any explanation for these facts other than that tumor deposits occluded the lymphatic channels.

These findings raise serious concerns regarding the utility of SLN mapping in patients with T3 tumors, as 
in a third of them, SLNs will not be marked and therefore this technique should not be used.

A fourth interesting point from this study is the SLN ratio (Table 3 ). In $\mathrm{T} 1$ tumors, we had to harvest 9 SLNs in order to diagnose 1 positive SLN (SLNs vs SLNs $(+)$ and this ratio decreased to 7.5 SLNs: $1 \mathrm{SLN}(+)$ in T2 tumors and to 1.6 SLNs: 1 SLN (+) in T3 tumors.

As previously stated, most of the experience gained until now regarding the use of sentinel node mapping has come from the Japanese literature and deals mainly with early gastric cancer [29]. Unfortunately, the prevalence of early gastric cancer in Israel, as in the rest of the Western countries, is very low. Most of the gastric cancer surgery done in our institute is for advanced disease and we believe that there is little place for SLN mapping in these cases, as shown by our results. Reviewing our database for gastric cancer in the past 10 years we found out that $61.8 \%$ of our patients were in stage 3 and 4 of their disease. It is logical to assume that the modern approach of neoadjuvant treatment for advanced-stage gastric cancer has some influence on the integrity of the lymphatic network not expected in a group of patients not managed by this approach. Therefore, we believe that SLN evaluation in this particular group of patients is futile.

Although in some studies it was considered sufficient to base the clinical treatment decision on the pathological evaluation of SLNs alone, and in so doing reduce expenses, in almost $10 \%$ of our patients there was a discrepancy between $\operatorname{SLNs}(-)$ and non $\operatorname{SLNs}(+)$, a factor that would create a bias in the selection of patients for adjuvant treatment. Therefore, we believe that, at least in our series, all LNs, SLNs and non SLNs, should be evaluated.

Based on our small series, it is difficult to formulate any valuable conclusion. Further studies, preferably multicentric, are required to gain knowledge on the subject of sentinel node mapping in gastric cancer patients. However, our study showed a high rate of accuracy when comparing SLN status and metastatic involvement of the lymph nodes, and SLN mapping may therefore be an additional instrument for planning the extent of lymphadenectomy. Nonetheless, SLN mapping for T3 tumors is futile, as in a third of the patients the evaluation of LN status would be misleading.

\section{References}

1. Cabanas RM. An approach for the treatment of penile carcinoma. Cancer 1977;39:456-66.

2. Morton DL, Wen DR, Wong JH, Economou JS, Cagle LA, Storm FK, et al. Technical details of intraoperative lymphatic mapping for early stage melanoma. Arch Surg 1992;127:392-9.
3. Chung MH, Ye W, Giuliano AE. Role for sentinel lymph node dissection in the management of large $(\geq 5 \mathrm{~cm})$ invasive breast cancer. Ann Surg Oncol 2001;8:688-92.

4. Cox CE, Pendas S, Cox JM, Joseph E, Shons AR, Yeatman T, et al. Guidelines for sentinel node biopsy and lymphatic mapping of patients with breast cancer. Ann Surg 1998;227: 645-53.

5. Medina-Franco H, Beenken SW, Heslin MJ, Urist MM. Sentinel node biopsy for cutaneous melanoma in the head and neck. Ann Surg Oncol 2001;8:716-9.

6. Reintgen D, Balch CM, Kirkwood J, Ross M. Recent advances in the care of the patient with malignant melanoma. Ann Surg 1997;225:1-14.

7. Kitagawa Y, Fujii H, Mukai M, Kubota T, Ando N, Watanabe M, et al. The role of sentinel lymph node in gastrointestinal cancer. Surg Clin North Am 2000;80:1799-809.

8. Thorn M. Lymphatic mapping and sentinel node biopsy: is the method applicable to patients with colorectal and gastric cancer? Eur J Surg 2000;166:755-8.

9. Wood TF, Saha S, Morton DL, Tsioulias GJ, Rangel D, Hutchinson W Jr, et al. Validation of lymphatic mapping in colorectal cancer: in vivo, ex vivo, and laparoscopic techniques. Ann Surg Oncol 2001;8:150-7.

10. Kim MC, Kim HH, Jung GJ, Lee JH, Choi SR, Kang DY, et al. Lymphatic mapping and sentinel node biopsy using 99mTC tin colloid in gastric cancer. Ann Surg 2004;239:383-7.

11. Song X, Wang L, Chen W, Pan T, Zhu H, Xu J, et al. Lymphatic mapping and sentinel node biopsy in gastric cancer. Am J Surg 2004:187:270-3.

12. Nimura $H$, Narimiya N, Mitsumori N, Yamazaki Y, Yanaga K, Urashima M. Infrared ray electronic endoscopy combined with indocyanine green injection for detection of sentinel nodes of patients with gastric cancer. Br J Surg 2004;91:575-9.

13. Pacelli F. Lymph node staging. 4th International Gastric Cancer Congress, New York, April 29-May 2, 2001. Abstract \#S27:325.

14. Aikou T, Higashi H, Natsugoe S, Hokita S, Baba M, Tako S. Can sentinel node navigation surgery reduce the extent of lymph node dissection in gastric cancer? Ann Surg Oncol 2001;9(Suppl): 90S-3S.

15. Catarci M, Guadagni S, Zaraca F, Pistoia MA, Mastracchio A, Trecca A, et al. Prospective randomized evaluation of preoperative endoscopic vital staining using $\mathrm{CH}-40$ for lymph node dissection in gastric cancer. Ann Surg Oncol 1998;5:580-4.

16. Miwa K. Optimal nodal dissection for early gastric cancer. Nippon Geka Gakkai Zasshi 2001;102:484-9.

17. Nimura H, Kashimura H, Sano Y, Aoki T, Fujisaki J, Narimiya $\mathrm{N}$, et al. Sentinel node navigation using an infrared electronic endoscope, and the possibility of expanding indications for gastrectomy preserving the hepatic and celiac branches of the vagus nerve. 4th International Gastric Cancer Congress, New York, April 29-May 2, 2001. Abstract \#547:594.

18. Osaka H, Yashiro M, Sawada T, Yamada S, Ogawa Y, Nakata B, et al. RT-PCR detection of gastric cancer cells in sentinel lymph nodes. 4th International Gastric Cancer Congress, New York, April 29-May 2, 2001. Abstract \#383:430.

19. Bilchik AJ, Saha S, Tsioulias GJ, Wood TF, Morton DL. Aberrant drainage and missed micrometastases: the value of lymphatic mapping and focused analysis of sentinel lymph nodes in gastrointestinal neoplasms. Ann Surg Oncol 2001;9(Suppl): 82S-85S.

20. Hiratsuka M, Miyashiro I, Ishikawa O, Furukawa H, Yamada T, Murata $\mathrm{K}$, et al. Is sentinel node biopsy feasible for T 1-2 gastric cancer? 4th International Gastric Cancer Congress, New York, April 29-May 2, 2001. Abstract \#006:29.

21. Ishizaki M, Kurita A, Kubo Y, Takashima S, Nishina T, Nishimura E. Evaluation of sentinel node identification with isosulfan blue in gastric cancer. Eur J Surg Oncol 2006;32:191-6.

22. Sobin LH, Wittekind C. TNM classification of malignant tumors. 6th ed. New York: John Wiley \& Sons; 2002. 
23. Kodama Y, Sugimachi K, Soejima K, Matsusaka T, Inokuchi K. Evaluation of extensive lymph node dissection for carcinoma of the stomach. World J Surg 1981;5:241-8.

24. Ichikura T, Chochi K, Sugasawa H, Yaguchi Y, Sakamoto N, Takahata R, et al. Individualized surgery for early gastric cancer guided by sentinel node biopsy. Surgery 2006;139:501-7.

25. McCulloch P, Nita ME, Kazi H, Gama-Rodriges J. Extended versus limited lymph nodes dissection technique for adenocarcinoma of the stomach. Cochrane Database Syst Rev 2004;4: CD001964.

26. Aikou T, Kitagawa Y, Kitajima M, Uenosono Y, Bilchik AJ, Martinez SR, et al. Sentinel lymph node mapping with GI cancer. Cancer Metastasis Rev 2006;25:269-77.
27. Iddings DM, Bilchik A. Gastric carcinoma: applying the sentinel node paradigm to improve the understanding of metastatic patterns and the possible role of selective lymphadenectomy. Ann Surg Oncol 2007;14:2432-4.

28. Gipponi M. Clinical applications of sentinel lymph-node biopsy for the staging and treatment of solid neoplasms. Minerva Chir 2005;60:217-33.

29. Lee JH, Ryu KW, Kim CG, Kim SK, Lee JS, Kook MC, et al. Sentinel node biopsy using dye and isotope double tracers in early gastric cancer. Ann Surg Oncol 2006;13:1168-74. 